\title{
Avaliação da efetividade da rede municipal pública de apoio diagnóstico em patologia clínica: o caso de Belo Horizonte (MG)
}

\author{
Effectiveness assessment of public clinical laboratories: \\ the case of Belo H orizonte, M inas Gerais State
}

Leyla Gomes Sancho ${ }^{1}$

José M uniz da Costa Vargens ${ }^{2}$

Rafael Gomes Sancho ${ }^{3}$

${ }^{1}$ Secretaria de Estado da Saúde de M inas Gerais. Rua Sapucaí 429, Floresta.

30150-050 Belo Horizonte M G. Isancho@uol.com.br

${ }^{2}$ Fundação Escola Nacional deSeguros.

${ }^{3}$ Universidade Federal de

Minas Gerais.
Abstract The organization of public clinical laboratories is experiencing changes without, however, an organizational assessment of its effective ness. Thestudy aimed to determinea parameter of effectiveness for public clinical laboratories of Belo H orizonte, M inas Gerais State, and set cut-off points for the sections of these laboratories. In order to do so, the total production and number of hours worked during a period of 7 months in the year 2008 were consolidated. Due to the entrance of the workers in the mode of production in the laboratories network, it could be observed a variability regarding the performance of these workers. The effectiveness parameter of the network was established in 29.90 tests per hour. Asa consequence of this first analysis, the cut-off points are: 15.50 for thehematology section; 67.29 for chemestry; 6.45 for parasitology; 11.35 for urinalysis; 4.94 for microbiology and 19.03 for immunology. From these results, it was concluded that the working process in laboratories can generate a decrease in effectiveness.

Key words Employee performance appraisal, Effectiveness, Organizational culture, Laboratory techniques and procedures
Resumo Importantes mudanças na organização de redes de laboratórios públicos de patologia clínica vêm ocorrendo sem, no entanto, uma decorrente avaliação de sua efetividade organizacional. 0 estudo pretendeu determinar um parâmetro da efetividade da redepública deapoio diagnóstico em patologia clínica do município de Belo Horizonte e determinar os seus pontos de corte para as seções desses laboratórios. Para tanto, se consolidou por seção e pelo conjunto dos laboratórios da redea produção total realizada e o número de horas trabaIhadas durante um período de setemeses no ano de 2008. Foi observada nas seções do conjunto dos laboratórios da rede, decorrente da inscrição dos trabalhadores no modo de produção do setor, uma variabilidade em relação ao desempenho do conjunto dos trabalhadores. 0 parâmetro de efetividade da rede foi estabelecido em 29,90 exames por hora e 0 ponto de corteassumido para as seções, como consequência da primeira análise, ficou assim estabelecido: 15,50 para hematologia, 67,29 para bioquímica, 6,45 para parasitologia, 11,35 para urinálise, 4,94 para microbiologia e 19,03 para sorologia. Concluiu-se que o processo de trabalho nos laboratórios pode gerar uma diminuição na sua efetividade, assim como, por decorrência, suscitou a necessidade da implementação da avaliação como um instrumento de gestão no setor.

Palavras-chave Avaliação de desempenho, Efetividade, Cultura organizacional, Técnicas e procedimentos de laboratório 


\section{I ntrodução}

Nas últimas décadas, vêm ocorrendo mudanças importantes no que tange à organização de redes delaboratórios públicos deapoio diagnóstico em patologia clínica. Mudanças advindas do decorrente incremento de grupos de ações desenvolvidos em cada nível de atenção à saúde e devido às características da população de abrangência sob a sua responsabilidade. Agrega-se ainda a essas a incorporação da automação no processamento dos exames.

$N$ as décadas de setenta e oitenta, o modelo organizacional estabelecido na rede pública erao do laboratório localizado na mesma área física do posto de saúde e/ou do centro de saúde, realizando uma pequena diversidadedeexames, processados manualmente.

Em meados da década de noventa do século $X X$, particularmente com a implantação do SistemaÚ nico deSaúde(SUS), a organização dos serviços de patologia clínica passa a ser orientada pela diretriz da hierarquização das ações - coerente e articulada com os demais serviços do SUS, em que se prevê a implantação de postos de coleta referenciados aos laboratórios distritais (ou regionais) e uma regulação da oferta, adequada às necessidades de saúde, subordinada à combinação de tecnologias diversificadas, adaptadas aos aspectos sociodemográficos, epidemiológicos, sanitários e econômicos de uma dada populaçãa ${ }^{1}$.

$\mathrm{N}$ a reestruturação do Sistema $\mathrm{N}$ acional de Laboratórios de Saúde Pública (SILAB), instituída em 1977 e depois readequada em 2004, organizada de forma hierarquizada por sub-redes, de acordo com agravos ou programas em consonância com as atividades relacionadas à vigilância à saúde, sanitária e assistência médica, propõe-se a formalização dos laboratórios locais (LL), os quais integram uma rede municipal de laboratórios de saúde pública, sendo de sua competência a realização de exames básicose essenciaiseo encaminhamento para o laboratório de referência municipal ou estadual das amostras inconclusivas, as para complementação de diagnóstico e aquelas destinadas ao controle de qualidade analítico, que respondem às necessidades programáticas da atenção primária/básica da atenção à saúde.

A centralização do processamento de exames na rede pública de laboratórios de patologia clínica, ao propiciar a aquisição de um número adequado de equipamentos automatizados, imprime um substancial aumento do rendimento dos equipamentose, por conseguinte, da produtividade dos recursos humanos, se comparado com esses mesmos equipamentos pulverizados em vários pequenos laboratórios.

A partir da automação em relação ao processamento deexames laboratoriais, impôs-se, como uma premissa, a aplicação do conceito de economia de escala no setor, que, como consequência, introduz uma nova prática em termos organizacionais. Isto, em termos econômicos, significa que a elasticidade custo-produção é menor do que a unidade, ou seja, os custos aumentam menos que o nível de produção?2.

A redepública deapoio diagnóstico em patologia clínica do município de Curitiba, no Paraná, foi uma das primeiras a efetivar a reorganização de sua rede na perspectiva de uma central única de processamento de exames. Sendo quea união das duas pontas do sistema - descentralização da coleta e a centralização do processamento - é dada a partir da implantação de um sistema logístico que recolhe as amostras biológicas nas unidades de saúde e as leva à central de processamento, cujo serviço no município está terceirizado para a Empresa Brasileira de Correios e Telégrafos (EBCT). Como decorrência desse processo, foi observada uma redução de custos, um aumento na qualidade e 0 encaminhamento do resultado do exame em tempo oportuno. Segundo Santos et al. ${ }^{3}$, o laboratório centralizado de Curitiba processava, nos anos noventa do século passado, aproximadamente dois milhões de exames por ano.

A rede de apoio diagnóstico em patologia clínica do município de Belo Horizonte

A rede municipal de apoio laboratorial de Belo H orizonte é composta por cinco laboratórios distritais, os quais realizam um cardápio de 62 tipos de exames - de acordo com os protocolos de atenção dos programas verticais do Sistema Municipal deSaúdee sob a perspectiva do cardápio sugerido para o Grupo A de exames pelo manual de orientação aos gestores sobre a organização da rede de laboratórios -, distribuídos em seis seções (hematologia, bioquímica, urinálise, parasitologia, microbiologia e sorologia), cuja composição de recursos humanos e material não guarda similaridade entre os distintos laboratórios. Vale citar que o município de Belo Horizonte é subdividido em nove distritos sanitários, os quais seguem a delimitação geográfica das administrações regionais do município.

A formalização da regionalização dos laboratórios distritais, em vista da não cobertura de um 
laboratório para cada distrito sanitário, ou meIhor, para uma dada população de abrangência, em alguma medida, obedeceu aos seguintes crité rios: acesso viário, tamanho da população das áreas de abrangência de cada distrito sanitário, número de Unidades Básicas de Saúde (UBS) em cada distrito sanitário e aproveitamento de áreas físicas já existentes. Ressalta-se que, com o processo demunicipalização, os extintos prontosatendimentos médicos (PAM) do Instituto Nacional de Assistência M édica e Previdência Social (INAM PS) foram absorvidos e com eles as suas áreas físicas afins, inclusive as dos laboratórios.

$\mathrm{Na}$ rede pública de laboratórios de patologia clínica do município de Belo H orizonte, a incorporação da automação, a partir do ano de 1996, agregada à centralização do processamento de exames em laboratórios distritais e implantação de um sistema de informação próprio, deflagrou um ganho em termos de economia de escala e também um aumento na oferta e na cobertura de exames necessários para responder à demanda em relação às ações programáticas da política municipal de saúde, bem como possibilitou avaliar a qualidade da execução dos exames eimplementar o planejamento, programação e regulação dessa atividade fim.

\section{Avaliação como subsídio}

0 processo de avaliação, embora em voga nos meios acadêmicos, ainda não encontrou de fato ressonância nos sistemas públicos de saúdet. Os sistemas de saúde, ainda hoje, se encontram em uma fase de consolidação da regulação e regulamentação da prestação de bens e/ou serviços à população sob sua responsabilidade, ou seja, na rede pública de atenção à saúde, ainda não se observa, como uma salutar decisão, bem como para uma salutar tomada de decisão, uma concomitante avaliação das ações de saúde disponibilizadas para a população. M esmo que no cenário nacional, particularmente no nível federal de governo, haja concreta proposta de avaliação de ações e de serviços implementados por um sistema de saúde $e^{5,6}$.

$\mathrm{N}$ a especificidade da avaliação em relação aos laboratórios de apoio diagnóstico em patologia clínica, os escassos estudos nacionais e, principalmente, os internacionais, se referem ao custo, à produtividade e à implantação de sistema de informação como os itens de interesse $e^{7,8}$.

Nesse sentido, após observar a não deflagração de qualquer ação de avaliação em relação à rede de apoio diagnóstico em patologia clínica da Secretaria M unicipal de Saúde de Belo Horizonte (SM SA-BH), procedeu-se, como uma primeira aproximação, a uma avaliação de desempenho dos laboratórios distritais, por seção, pertencentes à rede. Sendo que o intuito é o de referir um parâmetro da efetividadeem relação à redeper se, e, por conseguinte, estimar o possível ganho de oportunidade dos laboratórios distritais, os quais possam servir como subsídio para avaliar, de forma recorrente, a sua efetividade organizacional.

\section{M ateriais emétodos}

Para a realização da análise, em termos metodológicos, tomou-se como marco teórico o conceito de produtividade utilizado por $\mathrm{H}$ artz et al. ${ }^{9}$, em que a produtividade dos recursos profissionais a partir do modo como os recursos são usados para produzir serviços é medida em unidades físicas. $\mathrm{Ou}$, dito de outra forma, a capacidade de produção de um trabalhador, ajustada pela sua taxa de ocupação, que representa a taxa de sua jornada de trabalho dedicada à produção.

A análise de desempenho, por seção, de cada laboratório distrital foi efetivada a partir do mé todo de cálculo denominado produtividade de fator simples $(\mathrm{sfp})^{10}$, que considera a seguinte função: produção de exames no mês/total de horas trabalhadas no mês pelo conjunto dos trabalhadores em cada seção:

$\mathrm{Sfp}=\frac{\text { output do produto } \mathrm{A} \text { (quantidade do produto) }}{\text { input do recurso (unidade do recurso) }}$

A amostra considerada para a análise foi a produção realizada em quatro laboratórios distritais durante um período de sete meses e o número de dias trabalhados nos meses de janeiro a julho do ano de 2008. Sendo que, em janeiro, 0 número de dias trabalhados corresponde a 22 dias, no mês de fevereiro, 16 dias, em março, 20 dias, no mês abril, 21 dias e nos meses de maio, junho e julho, 20, 21 e 23 dias, respectivamente. Vale informar que o quinto laboratório distrital não foi considerado no estudo, por não se enquadrar nos critérios de inclusão. Os critérios de inclusão definidos foram os dados de produção validados e o recadastramento recente dos recursoshumanos - primeiro trimestre do ano de 2008.

0 período analisado (sete meses) levou em consideração os seguintes critérios: período escolar eo deférias escolares; cobertura em relação 
ao controle laboratorial da maioria das ações programáticas desenvolvidas na SM SA-BH e o quadro nosológico prevalente dasáreas deabrangência, que demanda exames de patologia clínica, aí incluído o período epidêmico da dengue (abril/maio).

Os dados primários relacionados à produção realizada nos laboratórios distritais foram coletados a partir de um processo manual efetuado em cada laboratório através de planilhas, as quais guardam similaridade com o modelo preconizado pelo sistema de informação do Sistema Ú nico de Saúde - SIGTAB. Isto porque o sistema informatizado dos laboratórios de patologia clínica (Sistema Laboratório Patologia Clínica - SLPC) consolida os dados relativos à produção a partir da demanda - coleta realizada no posto de coleta das unidades básicas. Ou seja, desconsidera as amostras biológicas encaminhadas para a rede (auxiliar) privada, as perdas de material biológico ou mesmo o reprocessamento de exames.

Para consolidar o número de horas trabaIhadas, em cada seção, nos distintos laboratórios distritais, tomou-se como subsídio a carga horária, em média, de cada servidor alocado na seção e o número de dias úteis no mês. Os dados relativos à carga horária dos profissionais foram coletados a partir do cadastro de recursos humanos da gerência de apoio diagnóstico da SM SA-BH.

O utras informações advieram de informantes-chave, tais como o modus operandi (processo de trabalho) dos laboratórios distritais; a ocorrência, ou não, de falta de insumos; o atraso, ou não, na entrega dos insumos e a manutenção adequada, ou não, dos equipamentos. A coleta dessas informações foi realizada a partir de entrevistas informais com servidores desses laboratórios. Vale citar que essas informações são de extrema importância, no sentido de subsidiar e, por conseguinte, validar os dados coletados e relativizar os resultados encontrados.

N esta medida, a primeira etapa da construção do modelo do estudo se ateve à formalização do desempenho por hora trabalhada, por mês, por laboratório distrital, a partir da consolidação da produção mensal por seção em cada laboratório distrital, seguido pela soma das cargas horárias diárias dispensadas pelos trabalhadores nas seções. A carga horária total por seção no mês foi obtida através do ajuste desta carga horária pelo número de dias úteis no mês.

A etapa seguinte se consubstanciou em imputar pontos de cortes para as seções no que tange ao desempenho dos profissionais, em que se utilizou para a sua formalização, a partir do mesmo conjunto de dados coletados, o seguinte cálculo: média das médias da produção por hora trabalhada por seção, por mês, do conjunto dos laboratórios no período estudado.

Em relação à formalização do parâmetro, entendido como um valor numérico querepresenta o limite superior observado (real) da análise, o somatório incluiu a produção total realizada e 0 total de horas trabalhadas de todos os laboratórios da rede no período. A partir deste (parâme tro) se inferiu um indicador que aponta a oportunidade de ganho ${ }^{11}$ para cada laboratório da rede. A oportunidade de ganho é representada por um delta que significa a diferença entre o val or possível de ser al cançado (parâmetro) e o valor encontrado para cada indicador sob comparação, sendo que a sua composição é dada pelo produto entre a diferença dos valores - parâmetro e indicador - e o número de horas trabal hadas referente ao indicador sob avaliação.

\section{Resultado}

A análise do desempenho dos recursos profissionais tem a produção realizada em um determinado período como a primeira variável aser conside rada. No caso dos laboratórios distritais, de agora em diante denominados de A, B, C e D, a produção por seção está demonstrada na Tabela 1.

Vale mencionar, até para melhor compreensão dos valores apresentados a seguir, que o laboratório distrital A cobre uma população de 408.000 habitantes, de classe socioeconômica mais favorecida, apresentando uma estrutura mais idosa ecom predomínio feminino, cujo índice de vulnerabilidade à saúde se situa no risco baixo e têm dezenove UBS a si agregadas. 0 laboratório distrital $B$ dá cobertura a uma população de 523.000 habitantes, cujo índice de vulnerabilidade à saúde se inscreve como sendo de risco médio eéreferência de 34 UBS. 0 laboratório distrital $C$ atua sobre uma população de abrangência de 337.351 habitantes, se enquadra como sendo de risco médio no que se refere ao índice de vulnerabilidade à saúde e também têm dezenove U BS sob sua responsabilidade. Já o laboratório D tem 35 U BS como referência e cobreuma população de 534.000 habitantes, mais desfavorecida em termos socioeconômicos, com perfil mais jovem e com índice de vulnerabilidade à saúde de risco elevado. Ressalta-se que o índice devulnerabilidadeà saúde éuma medida utilizada pela gerência de assistência à saúde da Secre- 
taria Municipal de Saúde e pode ser mais bem apropriada no Plano Municipal de Saúde do pe ríodo 2005 a 2008.

Chama a atenção a produção efetivada nos distintos laboratórios distritais que, mesmo estruturados a partir de uníssona diretriz, apresentam val ores dissonantes entre as diferentes seções, e, por conseguinte, dos correspondentes tipos de exames realizados em cada seção, sugerindo, por exemplo, que há uma demanda diferenciada, a qual pode ser decorrente, como uma hipótese, de inerente condição socioeconômica da população decada área de abrangência do município, configurada, inclusive, por diferentes prevalências ou mesmo por diferente quadro nosológico. Ressalta-se, no entanto, que essa discussão não faz parte do objetivo explícito deste estudo, mas serve como um elemento para reflexões. Também chama a atenção o fato da produção apresentar em alguns meses e em al gumas seções valores abaixo do habitualmente praticado, sugerindo a ocorrência de falta de insumos ou problemas de manutenção dos equipamentos.

A segunda variável dimensionada no queconcerneà análise foi relativa ao quadro de recursos humanos alocado em cada laboratório distrital e o número de horas trabalhadas por semana (carga horária contratual), por seção, pelo conjunto dos profissionais. A carga horária do quadro de recursos humanos se apresenta conforme a Tabela 2.

0 total de horas trabal hadas no conjunto dos laboratórios no período estudado foi de 85419,5 horas.

O número de profissionais alocados nas seções nos distintos laboratórios distritais, assim como a carga horária praticada, não é equivalente. Chama a atenção, por exemplo, o número de trabalhadores na seção de parasitologia do laboratório D e a correspondente carga horária total, significando que um grupo de trabalhadores cumpre uma carga horária menor do que a contratual na seção, ou melhor, reforçando a prática da partição da carga horária entre as diversas seções. Ressalta-se que, na SM SA-BH, os bioquímicos têm contratos de vinte ou quarenta horas semanais (extensão de jornada) e os técnicos de laboratório, em geral, contrato de trinta horas semanais. Também é importante ressaltar que ocorre nestes laboratórios, como na maio-

Tabela 1. Produção por seção por laboratório distrital nos meses de janeiro a julho de 2008.

\begin{tabular}{lcccccrr}
\hline & Hematologia & Bioquímica & Parasitologia & Microbiologia & Urinálise & Sorologia & Total \\
\hline Lab. A & 42467 & 186992 & 14194 & 11809 & 22145 & 8928 & 286535 \\
Lab. B & 77197 & 334918 & 29382 & 18486 & 39455 & 20455 & 519893 \\
Lab. C & 52458 & 228600 & 20379 & 13972 & 50388 & 14420 & 806428 \\
Lab. D & 86487 & 330469 & 33915 & 26939 & 48059 & 12026 & 537895 \\
Total & 258609 & 1080979 & 97870 & 71206 & 160047 & 55829 & 1724540 \\
\hline
\end{tabular}

Fonte: Laboratórios distritais da gerência de apoio diagnostico da SM SA-BH.

Tabela 2. Quantitativo de recursos humanos e carga horária semanal por seção por laboratório distrital.

\begin{tabular}{|c|c|c|c|c|c|c|c|c|c|c|c|c|}
\hline $\begin{array}{l}\text { Labc } \\
\text { distr }\end{array}$ & $\begin{array}{l}\cdot \frac{0}{0} \\
\frac{0}{0} \\
\frac{0}{0} \\
\frac{0}{1} \\
1\end{array}$ & 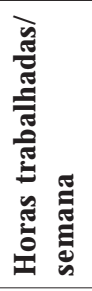 & $\begin{array}{l}\frac{\delta}{\varepsilon} \\
\frac{\delta}{\bar{\sigma}} \\
\frac{0}{0}\end{array}$ & 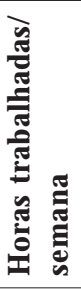 & $\begin{array}{l}\frac{0}{\overline{0}} \\
\frac{0}{0} \\
\frac{1}{\bar{n}} \\
\frac{0}{0} \\
0\end{array}$ & 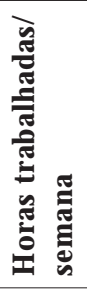 & 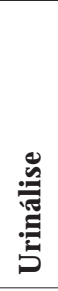 & 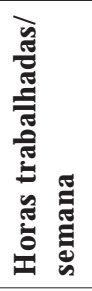 & $\begin{array}{l}\frac{0}{\overline{0}} \\
\frac{0}{0} \\
\frac{0}{0} \\
\frac{0}{\Sigma} \\
\frac{0}{2}\end{array}$ & 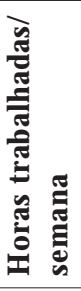 & $\begin{array}{l}\frac{\sigma}{\bar{\delta}} \\
\frac{\delta}{0} \\
\frac{0}{0}\end{array}$ & 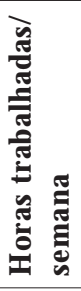 \\
\hline A & 6 & 180 & 5 & 160 & 6 & 150 & 7 & 145 & 6 & 95 & 1 & 20 \\
\hline B & 7 & 200 & 4 & 150 & 5 & 130 & 4 & 90 & 5 & 120 & 3 & 70 \\
\hline C & 3 & 80 & 3 & 90 & 7 & 87 & 6 & 90 & 3 & 100 & 2 & 13 \\
\hline D & 9 & 167,5 & 8 & 180 & 16 & 177,5 & 12 & 207,5 & 11 & 188 & 2 & 35 \\
\hline
\end{tabular}

Fonte: Setor de apoio diagnóstico da SM SA-BH. 
ria dos serviços públicos em todo o país, o desvio de função, o que significa a ocorrência de alocação de trabalhadores de outras categoriais profissionais nos laboratórios, ou mesmo, diante do processo de municipalização dos recursos humanos, uma mesma categoria profissional, de acordo com a instituição de origem, desempenhar distinta carga horária.

0 desempenho do conjunto dos trabalhadores, por seção, nos laboratórios distritais da rede de apoio diagnóstico da Secretaria Municipal de Belo Horizonte (SM SA-BH), considerando as duas variáveis - produção mensal por seção e total de horas trabal hadas pela equipe na seção no mês - pode ser visualizado na Tabela 3.

0 resultado, no entanto, não traduz o desempenho per se de cada profissional por hora trabalhada. Para esse tipo de resultado, seria necessária uma avaliação adicional, in loco, do processo de trabalho do conjunto dos trabalhadores entre as seções dos laboratórios distritais durante um determinado período.

Conforme demonstrado na Tabela 3, e corroborando a informação de que os profissionais fazem diariamente, em maior ou menor grau, rodízio entre as seções no laboratório, o seu de

Tabela 3. Desempenho dos recursos humanos nos laboratórios distritais da rede no período.

\begin{tabular}{|c|c|c|c|c|c|c|c|c|}
\hline \multicolumn{9}{|c|}{$\begin{array}{l}\text { Seções M eses } \\
\text { M }\end{array}$} \\
\hline & \multicolumn{8}{|c|}{ Lab. Distrital A } \\
\hline & jan & fev & mar & $a b r$ & maio & jun & julh & M édia \\
\hline Hematologia & 7,62 & 8,29 & 7,97 & 9,51 & 7,93 & 8,59 & 7,17 & 8,15 \\
\hline Bioquímica & 35,21 & 41,08 & 43,38 & 40,76 & 43,80 & 39,90 & 38,79 & 40,42 \\
\hline Parasitologia & 3,32 & 3,35 & 3,76 & 3,65 & 2,87 & 3,20 & 2,75 & 3,27 \\
\hline Urinálise & 4,90 & 5,57 & 5,55 & 5,49 & 4,96 & 5,58 & 4,91 & 5,28 \\
\hline M icrobiologia & 3,67 & 4,31 & 4,14 & 5,46 & 4,10 & 5,83 & 4,96 & 4,64 \\
\hline \multirow[t]{3}{*}{ Sorologia } & 14,06 & 17,32 & 16,60 & 15,36 & 13,94 & 15,71 & 15,12 & 15,44 \\
\hline & \multicolumn{8}{|c|}{ Lab. Distrital B } \\
\hline & jan & fev & mar & $\mathrm{abr}$ & maio & jun & julh & \\
\hline Hematologia & 11,36 & 12,81 & 12,77 & 15,38 & 14,65 & 14,59 & 11,81 & 13,34 \\
\hline Bioquímica & 73,09 & 83,65 & 83,80 & 82,32 & 68,15 & 77,73 & 71,75 & 77,21 \\
\hline Parasitologia & 8,06 & 9,03 & 8,73 & 8,98 & 6,66 & 6,39 & 6,94 & 7,83 \\
\hline Urinálise & 11,97 & 13,17 & 13,08 & 13,22 & 11,34 & 12,66 & 11,35 & 12,40 \\
\hline M icrobiologia & 5,37 & 6,43 & 5,15 & 6,01 & 3,22 & 5,76 & 5,29 & 5,32 \\
\hline \multirow[t]{3}{*}{ Sorologia } & 10,77 & 11,03 & 9,86 & 10,35 & 9,25 & 10,09 & 9,32 & 10,09 \\
\hline & \multicolumn{8}{|c|}{ Lab. Distrital C } \\
\hline & jan & fev & mar & $\mathrm{abr}$ & maio & jun & julh & \\
\hline H ematologia & 21,13 & 21,07 & 24,75 & 27,25 & 23,80 & 23,09 & 17,67 & 22,68 \\
\hline Bioquímica & 78,28 & 86,32 & 96,99 & 98,30 & 83,91 & 90,18 & 80,35 & 87,76 \\
\hline Parasitologia & 7,90 & 9,22 & 9,21 & 10,13 & 6,29 & 6,05 & 7,89 & 8,10 \\
\hline Urinálise & 19,16 & 22,08 & 22,16 & 24,07 & 20,92 & 20,44 & 8,00 & 19,55 \\
\hline M icrobiologia & 5,02 & 5,73 & 5,89 & 5,93 & 4,99 & 1,88 & 4,50 & 4,85 \\
\hline \multirow[t]{3}{*}{ Sorologia } & 41,99 & 37,14 & 48,08 & 48,02 & 37,38 & 40,92 & 16,39 & 38,56 \\
\hline & \multicolumn{8}{|c|}{ Lab. Distrital D } \\
\hline & jan & fev & mar & $a b r$ & maio & jun & julh & \\
\hline Hematologia & 16,52 & 17,54 & 17,81 & 19,86 & 17,68 & 18,42 & 16,92 & 17,82 \\
\hline Bioquímica & 58,17 & 72,03 & 70,13 & 71,24 & 61,67 & 65,66 & 47,51 & 63,77 \\
\hline Parasitologia & 6,63 & 7,45 & 7,22 & 7,92 & 5,48 & 5,61 & 5,97 & 6,61 \\
\hline Urinálise & 7,65 & 8,66 & 8,38 & 8,81 & 7,72 & 8,13 & 7,72 & 8,15 \\
\hline M icrobiologia & 4,67 & 5,47 & 5,34 & 5,47 & 3,79 & 5,19 & 4,74 & 4,95 \\
\hline Sorologia & 11,40 & 14,17 & 13,55 & 14,86 & 13,68 & 12,28 & 4,24 & 12,03 \\
\hline
\end{tabular}

Fonte: Setor de apoio diagnóstico e laboratórios distritais da SM SA-BH. 
sempenho por hora trabalhada é menor onde ocorre, em maior grau, a partição da carga horária em um número pequeno de horas trabal hadas em cada seção. Este fato fica patente ao se observar os resultados dos laboratórios distritais C e D. No laboratório distrital C, o número de trabalhadores por seção é menor, a rotatividade entre as seções é menor e a produção por hora trabalhada é maior em todas as seções, diferente do laboratório $D$, que apresenta resultado oposto. Assim como se pode afirmar que, na seção da sorologia, ainda tomando os dois laboratórios como exemplo, onde a média de horas trabalhadas é de 17,5 no laboratório $D$ e de 23,3 no laboratório $C$ ea produção por hora de 12,03 e 38,56, respectivamente, a média da produção por hora nessa seção, do laboratório $D$, poderia chegar a 28,96, caso se considere, por equivalência, os valores do laboratório $\mathrm{C}$ em relação às horas trabalhadas.

Tomando os mesmos dados advindos da coleta, pode-se imputar os seguintes pontos de corte (média) em relação ao desempenho por trabalhador, por seção, para a rede de laboratórios do município de Belo Horizonte, considerando que a demanda por diferentes tipos de exames não guarda dependência entre si, ou seja, a ocorrência, em termos estatísticos, de uma distribuição normal em relação à demanda de exames.

Diante dos valores encontrados como ponto de corte para a rede de laboratórios da SM SABH (Tabela 4) e os valores apresentados na Tabela 3, correspondentes aos distintos laboratórios, é possível averiguar os que estão acima ou abaixo do valor indicado como referência. $\mathrm{Na}$ seção da hematologia, por exemplo, os laboratórios A e B apresentam valores abaixo do indicado, assim como na seção da urinálise, os laboratórios $\mathrm{B}$ e C apresentam valores acima da referência. Esta análise pode ser realizada em relação às demais seções dos distintos laboratórios, 0 que denota os desvios de um padrão da efetividade esperada.

0 parâmetro da efetividade da rede de laboratórios da Secretaria M unicipal deSaúde deBelo H orizonte é de 29,90 exames/hora - conforme 0 Gráfico 1, que correspondente ao valor do limite superior resultante da análise final. 0 valor de limite inferior, 20 exames/hora, é representado pela média. Ressalta-se que o valor do parâmetro é um indicativo preliminar, tendo em vista queo ideal para esse tipo de análise seria o de sua composição a partir deuma sériehistórica econsiderando alguns critérios como, por exemplo, a distribuição similar de equipamentos automatizados entre os laboratórios.

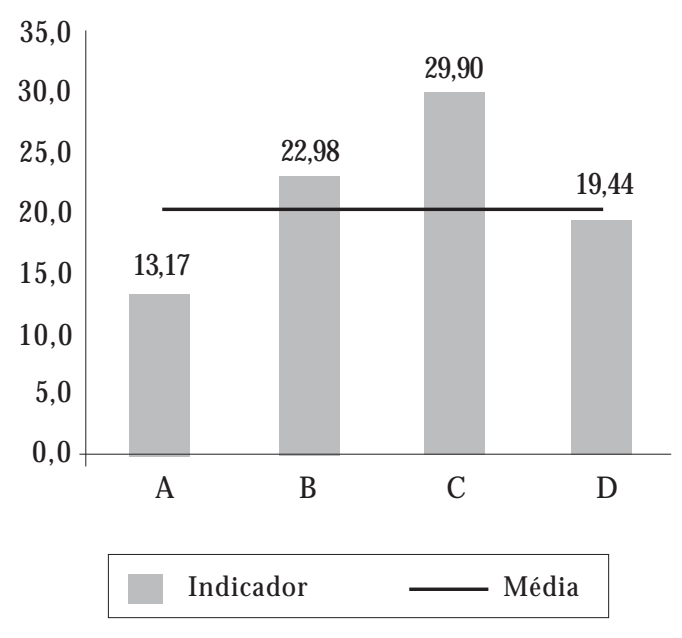

Gráfico 1. Parâmetro da efetividade da rede de laboratórios públicos do município de Belo Horizonte.

Fonte: Gerência de apoio diagnóstico da SM SA-BH.

Tabela 4. Pontos de corte da efetividade por seção para a rede de laboratórios da SM SA-BH.

\begin{tabular}{lrrrrrrrrrr}
\hline \multicolumn{1}{c}{ Seções } & Lab. A & Lab. B & Lab.C & Lab. D & Total & Média & $\begin{array}{c}\text { Desvio } \\
\text { padrão }\end{array}$ & $\begin{array}{c}\text { I.C. } \\
(95 \%)\end{array}$ & $\begin{array}{r}\text { Limite } \\
\text { mínimo }\end{array}$ & $\begin{array}{c}\text { Limite } \\
\text { máximo }\end{array}$ \\
\hline Hematologia & 8,15 & 13,34 & 22,68 & 17,82 & 61,99 & 15,50 & 6,21 & 6,08 & 9,29 & 21,71 \\
Bioquímica & 40,42 & 77,21 & 87,76 & 63,77 & 269,16 & 67,29 & 20,43 & 20,02 & 46,86 & 87,72 \\
Parasitologia & 3,27 & 7,83 & 8,10 & 6,61 & 25,81 & 6,45 & 2,22 & 2,17 & 4,24 & 8,67 \\
U rinálise & 5,28 & 12,40 & 19,55 & 8,15 & 45,38 & 11,35 & 6,20 & 6,08 & 5,14 & 17,55 \\
Microbiologia & 4,64 & 5,32 & 4,85 & 4,95 & 19,76 & 4,94 & 0,29 & 0,28 & 4,65 & 5,22 \\
Sorologia & 15,44 & 10,09 & 38,56 & 12,03 & 76,12 & 19,03 & 13,21 & 12,94 & 5,83 & 32,24 \\
\hline
\end{tabular}

Fonte: Gerência deapoio diagnóstico da SM SA-BH. 
N essa medida, a oportunidade de ganho dos laboratórios A, B e D - produção adicional possível - ao mês, pode chegar a, respectivamente, 51.970 exames no laboratório distrital A, 22.349 exames no laboratório distrital B e 41.387 exames no laboratório distrital $D$.

\section{Discussão}

0 resultado do estudo suscita uma série de discussões no que tange à organização da rede de apoio laboratorial no âmbito municipal e à padronização dos procedimentos, à importância da implantação de um sistema de informação que realmentesubsidiea gerência/gestão deum sistema de apoio diagnóstico no nível do município, à demanda de cada laboratório distrital e, principalmente, à interferência do modusoperandi dos distintos laboratórios e ao resultado do seu desempenho na perspectiva da construção de um parâmetro para avaliar a efetividade organizacional da rede, entendendo-se que a efetividade organizacional, segundo Campbell ${ }^{12}$, éo grau no qual cada um dos objetivos finais da organização é realizado sob certas restrições. Restrições que podem ser explicadas, em grande medida, pela análise dos processos produtivos no interior de uma organização, que traz no cerne a ação do trabalhador em relação introspectiva, com os usuários e com o processo organizacional. Entendendo-se que, no interior das organizações, há fluxos contínuos entre os atores/sujeitos nos seus espaços de produção em seus diferentes processos de trabalho' ${ }^{13}$.

A produção realizada nos distintos laboratórios distritais, se balizada com o quantitativo de recursos humanos, que varia de 25 a 45 profissionais em termos absolutos, denota que há uma correlação positiva entre as duas variáveis. No entanto, ao se proceder a análise do desempenho desses mesmos profissionais, a correlação énegativa, ou seja, o laboratório com maior número de profissionais é um dos que apresenta o mais baixo desempenho.

Estudo realizado por Valenstein et al. ${ }^{14} \mathrm{em}$ um total de 151 laboratórios de patologia clínica associados ao College of American Pathologists refere um resultado semelhante ao encontrado na rede de laboratórios da SM SA-BH. Segundo os autores, mesmo com a padronização de métodos, há uma variação no nível (qualiquantitativo) de recursos humanos alocados em uma determinada seção nos diferentes laboratórios da amostra, assim como há uma variação em ter- mos de desempenho entre as seções inter e intra laboratório.

No entanto, a determinação para o desempenho que envolve - como é o caso de um laboratório de apoio em patologia clínica - todos os recursos de um determinado produto, esta é efetivada através da combinação das diversas produtividades (de cada recurso) em uma única expressão - produtividade de fator total (tfp), a qual busca encontrar um resultado mais consistente do ponto de vista da avaliação global.

0 ideal neste estudo teria sido agregar ao modelo o rendimento dos equipamentos, resultante da combinação do número de equi pamentos e da sua especificação técnica (número de amostras que cada um é capaz de processar em um lote, tempo e número de homens/hora necessários para o seu processamento e perfil do profissional exigido para a sua operação), onde então

$$
\operatorname{tfp}=\frac{v}{\left(\Sigma \mathrm{A}_{\mathrm{i}} \mathrm{X}_{\mathrm{i}}\right)},
$$

sendo $i=1,2, \ldots n ; v=$ nível de atividade de produção; $A i$ = total de produção empregada eXI = coeficiente de ponderação associado a $\mathrm{Ai}^{10}$, assim como o balizamento desta produtividade com a apropriação dos custos dos inputs.

De acordo com M ugnol e Ferraz ${ }^{8}$, a automação e a informatização são fatores que alteram 0 ambiente laboratorial, tornando-os mais produtivo, mais eficiente e mais controlado. Ainda na opinião dos autores, é preciso avaliar de forma simultânea a produtividade, a qualidade, os custos, não sendo mais aceitável dar prioridade apenas aos dois primeiros sem levar em consideração a terceira variável.

Tomando como verdade a afirmação dos autores acima referida, cita-se a deficiência desse estudo, a de não incorporar dados referentes aos custos da produção dos exames nos laboratórios distritais. No entanto, essa é uma deficiência da maioria do sistema público de saúde, a de não incorporar a análise de custos como um instrumento de gestão $0^{15}$.

A implantação da distritalização/regionalização dos laboratórios propiciou não só a reorganização da rede de laboratórios do município, com a submissão desses a uma área de abrangência populacional, como também permitiu, diante de racional incorporação tecnológica concentração de equipamentos automatizados e informatizados -, a ampliação do seu escopo, ganho de economia de escala e a sua responsivi- 
dade. Ampliação que respondeu não só às necessidades dos protocolos assistenciais, mas também à cobertura da população em relação à atenção à saúde. Conforme Young e $\mathrm{McC}$ arthy ${ }^{16}$, ao se referirem sobre a introdução da integração de laboratórios de patologia clínica nos Estados Unidos (Legacy Health System), após a percepção dos nós críticos relacionados à pulverização de laboratórios (circulação das amostras biológicas, o sistema de informação e a padronização dos procedimentos), a regionalização imputou um ganho na qualidade de processamento e re sultado dos exames.

0 sistema próprio de informação da rede de laboratórios distritais do município de Belo Horizonte (SLPC) ainda é deficiente e necessita de uma série de ajustes, inclusive para subsidiar estudos como 0 ora apresentado. Isto porque, conforme já explicitado em relação a este estudo, uma gama de dados foi coletada manualmente.

0 déficit, no entanto, não se restringe a ape nas esse fato, ou seja, o de sequer responder às necessidades de cunho administrativo, mas principalmente por não permitir a concretização de avaliações mais pertinentes no que concerne à atenção coletiva e propiciar, na perspectiva individual da atenção, uma resposta mais ágil.

\section{Considerações finais}

O valor dimensionado para o parâmetro da efetividade da rede e os decorrentes para a oportunidade de ganho dos laboratórios distritais de vem, entretanto, ser balizados com discernimento e cautela. Discernimento no sentido que o serviço ora avaliado, mesmo que propício ao ganho de economia de escala, não deve incorrer na formalização de uma demanda induzida pela ofer- ta, e cautela no sentido que outras variáveis intervenientes (independência/dependência de processamento de exames nas seções, desabastecimento, absenteísmo, equipamento danificado, etc.) podem mudar os valores per se.

M esmo que haja uma correlação entre os valores referentes à produção, número de recursos humanos alocados, número de UBS atreladas e uma condizente população de abrangência a ser coberta, o processo de trabalho em pelo menos um dos laboratórios distritais da rede municipal de apoio diagnóstico em patologia clínica (laboratório D) apresenta uma perda na sua oportunidade de ganho. Fato possivelmente decorrente da utilização inadequada de intenso rodízio dos servidores entre as seções, o que, em grande medida, configura um tempo morto ${ }^{17} \mathrm{em}$ relação ao desempenho de seus profissionais. Como também éjustificada a perda de ganho do laboratório distrital A, por ter um potencial de oferta maior do que a da demanda referenciada.

A busca por padrões de efetividade para balizar a gestão dos laboratórios públicos leva à de terminação de pontos de desempenho possível. Recomenda-se, nessa medida, que para estudos futuros sejam envidados esforços na construção de um modelo que dimensione uma aproximação mais efetiva da capacidade potencial de produção dos laboratórios da rede municipal de apoio diagnóstico.

De qualquer sorte, o resultado desse estudo, mesmo que parcial e de forte cunho indicativo, tem ainda como objetivo, subjacente, trazer para o sistema público de saúde, e por decorrência para o setor público de apoio diagnóstico em patologia clínica, um alerta em relação à responsabilização no que tange à necessidade de se incorporar a avaliação como um instrumento eficaz de gestão.

\section{Colaboradores}

LG Sancho trabalhou na concepção teórica do estudo, análise dos dados e redação final do texto. JMC Vargens e RG Sancho trabalharam na consolidação e estatística dos dados. 


\section{Referências}

1. Brasil. M inistério da Saúde. Secretaria de Assistência à Saúde. Departamento de Descentralização da Gestão à Assistência. M anual de apoio aos gestores do SUS: organização da rede de laboratórios clínicos. N ormas e M anuais técnicos. [site da Internet] [acessado 2008 out]. Disponível em: http://www.bvsms. saude.gov.br/bvs/publicacoes/apoio_sus.pdf

2. Varian HR. Microeconomia: princípios básicos. 2ª ed. Rio de Janeiro: Campus; 1994.

3. Santos CA, Leonardi LJ, Voss STZ, Ito TS. O sistema de apoio diagnóstico terapêutico no nível local. In: Mendes EV, organizador. A organização da saúde no nível local. São Paulo: Hucitec; 1998.

4. Felisberto E. Da teoria à formulação de uma Política Nacional de Avaliação em Saúde. Cien Saude Colet 2006; 11(3):553-563.

5. Brasil. M inistério da Saúde. Secretaria de Atenção Básica. Planos Estaduais para fortalecimento das A ções de M onitoramento e Avaliação da Atenção Básica Diretrizes e O rientações, 2004. [site da Internet] [acessado 2008 out]. Disponível em: http://dtr2001. saude.gov.br/sas/PORTARIAS/Port2004/GM/GM 588.htm

6. Brasil. Ministério da Saúde. Secretaria de Atenção à Saúde. Departamento de Regulação, Avaliação e Controle de Sistemas. Coordenação Geral de Regulação e Avaliação. Programa Nacional de Avaliação de Serviços de Saúde, 2004. [site da Internet] [acessado 2008 out] Disponível em: http://pnass.datasus.gov.br/ documentos/CADERNO_PNASS.pdf

7. Valenstein PN, Praestgaard AH, Lepoff RB. Six-Year Trends in Productivity and Utilization of 73 Clinical Laboratories. Arch Pathol Lab Med 2001; 125(9):1153-1161.

8. Mugnol KCU, Ferraz M B. Sistema de Informação como ferramenta de cálculo e gestão de custos em laboratórios de análises clínicas. J Bras Patol M éd Lab 2006; 42(2):95-102.

9. Hartz ZM A, organizadora. Avaliação em Saúde: dos modelos conceituais à prática na análise da implantação de programas. Rio de Janeiro: Fiocruz; 1997.
10. Severiano Filho C, Dunda M FE, Batista GB. Análise das abordagens sobre medidas de produtividade. [site da Internet] [acessado 2008 out]. Disponível em: http://www.libdigi.unicamp.br/document/ ?down $=65$

11. Campos VF. Gerenciamento pelas diretrizes. Belo Horizonte: Fundação Christiano Ottoni; 1996.

12. Campbell JP. On the nature of organizational effectiveness. In: Goodman PS, Pennings JM, editors. New Perspectives on Organizational Effectiveness. San Francisco: Jossey-Bass; 1977. p. 13-55.

13. Franco TB, M erhy EE. M apas analíticos: um olhar sobre a organização e seus processos de trabalho. [site da Internet] [acessado 2009 mar 08]. Disponível em: http://www.hucff.ufrj.br/micropolitica/texto/ mapa_analitico.pdf

14. Valenstein PN, Souers R, Wilkinson DS. Staffing benchmarks for clinical laboratories: a College of American Pathologists Q-probes study of staffing at 151 institutions. Arch Pathol Lab M ed 2005; 129:467473.

15. Sancho LG, Vargens JM. Avaliação Econômica em Saúde na esfera de atenção local à saúde. Cien Saude Colet 2009; 14(supl.1):1513-1525.

16. Young DW, McCarthy SM. M anaging integrated delivery systems: a framework for action. Chicago: $\mathrm{He}$ alth Administration Press; 1999.

17. Dal Poz R, Pierantoni CR, Varella TC. Produtividade e Desempenho dos Recursos Humanos nos Serviços de Saúde. [site na Internet] [acessado 2008 nov]. Disponível em: seminario-brasilia.tripod.com/sitebuilder content/sitebuilderfiles/incentivos.pdf

Artigo apresentado em 17/11/2008

Aprovado em 19/02/2009

Versão final apresentada em 26/03/2009 